\title{
Structure
}

\section{Need for Speed: Examining Protein Behavior during CryoEM Grid Preparation at Different Timescales}

\section{Graphical Abstract}

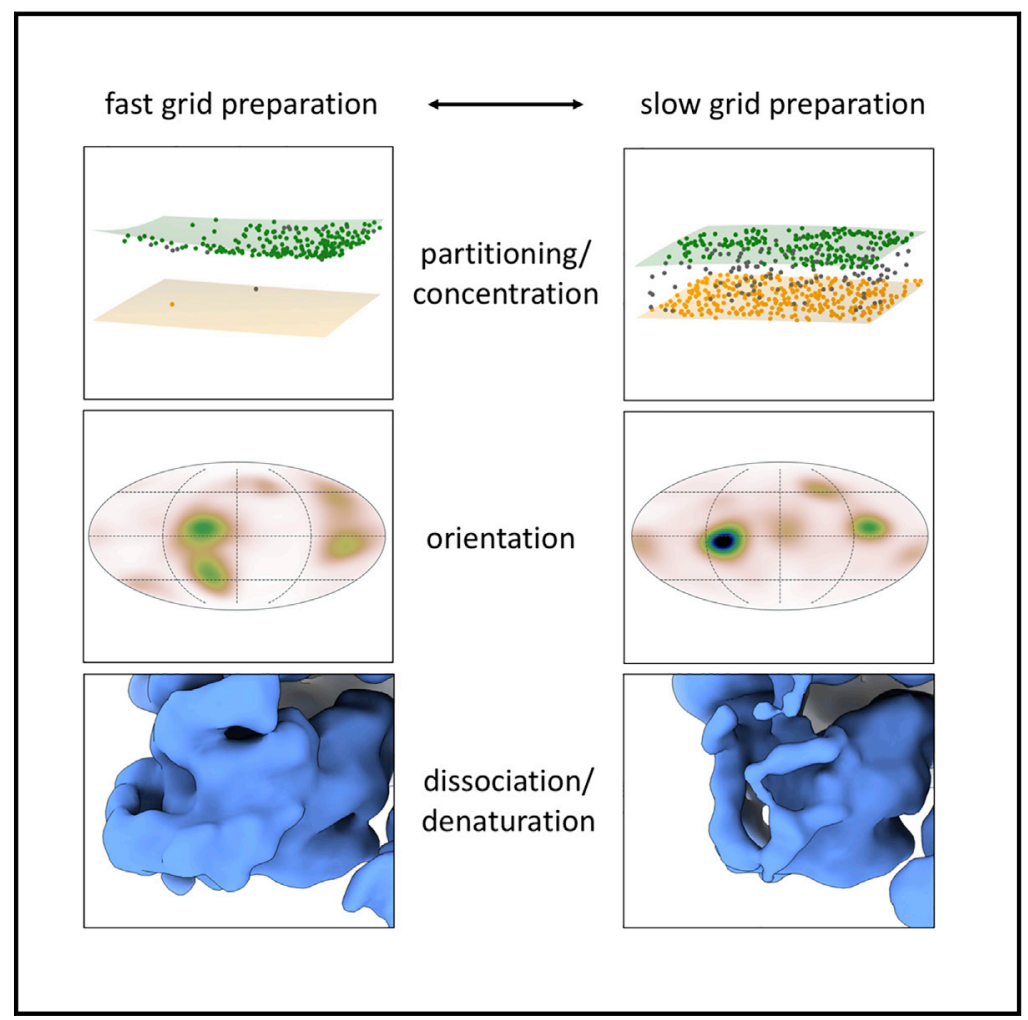

\section{Highlights}

- Particles partition to the air-water-interface even during fast (ms) grid making

- Higher grid-making speeds can result in greater angular spread of the specimen

- Higher grid-making speeds may reduce particle damage and subunit dissociation

- Different grid-making apparatus can affect sample concentration in the ice

\section{Authors}

David P. Klebl, Molly S.C. Gravett, Dimitrios Kontziampasis, ...,

Michele C. Darrow,

Rebecca F. Thompson,

Stephen P. Muench

\section{Correspondence}

r.f.thompson@leeds.ac.uk (R.F.T.), s.p.muench@leeds.ac.uk (S.P.M.)

\section{In Brief}

Here, we take advantage of new gridmaking procedures to investigate the effect of speed in cryoEM grid production on protein particle behavior. These results allow us to better understand how different grid-making approaches can affect the resultant grid with respect to particle damage, preferred orientation, and resultant concentration. 


\title{
Structure
}

\section{Need for Speed: Examining Protein Behavior during CryoEM Grid Preparation at Different Timescales}

\author{
David P. Klebl, ${ }^{1}$ Molly S.C. Gravett, ${ }^{2}$ Dimitrios Kontziampasis,,${ }^{1,3,4}$ David J. Wright, ${ }^{5}$ Robin S. Bon, ${ }^{5}$ Diana C.F. Monteiro, ${ }^{6}$ \\ Martin Trebbin, ${ }^{6,7}$ Frank Sobott, ${ }^{2,8}$ Howard D. White, ${ }^{9}$ Michele C. Darrow, ${ }^{10}$ Rebecca F. Thompson,, ${ }^{2, *}$ \\ and Stephen P. Muench ${ }^{1,11 \text {,* }}$ \\ ${ }^{1}$ School of Biomedical Sciences, Faculty of Biological Sciences \& Astbury Centre for Structural and Molecular Biology, University of Leeds, \\ Leeds LS2 9JT, UK \\ ${ }^{2}$ School of Molecular and Cellular Biology, Faculty of Biological Sciences \& Astbury Centre for Structural and Molecular Biology, University of \\ Leeds, Leeds LS2 9JT, UK \\ ${ }^{3}$ School of Chemical and Process Engineering, Faculty of Engineering and Physical Sciences, University of Leeds, Leeds LS2 9JT, UK \\ ${ }^{4}$ Institute of Business, Industry \& Leadership, University of Cumbria, Carlisle CA1 2HH, UK \\ ${ }^{5}$ School of Medicine, Faculty of Medicine and Health \& Astbury Centre for Structural and Molecular Biology, University of Leeds, Leeds, UK \\ 6Hauptman-Woodward Medical Research Institute, Buffalo, NY, USA \\ ${ }^{7}$ Department of Chemistry, State University of New York at Buffalo, Buffalo, NY, USA \\ ${ }^{8}$ Department of Chemistry, Biomolecular \& Analytical Mass Spectrometry Group, University of Antwerp, Antwerp, Belgium \\ ${ }^{9}$ Department of Physiological Sciences, Eastern Virginia Medical School, Norfolk, VA, USA \\ 10SPT Labtech, Melbourn Science Park, Melbourn, Cambridge, UK \\ ${ }^{11}$ Lead Contact \\ *Correspondence: r.f.thompson@leeds.ac.uk (R.F.T.), s.p.muench@leeds.ac.uk (S.P.M.) \\ https://doi.org/10.1016/j.str.2020.07.018
}

\section{SUMMARY}

A host of new technologies are under development to improve the quality and reproducibility of cryoelectron microscopy (cryoEM) grid preparation. Here we have systematically investigated the preparation of three macromolecular complexes using three different vitrification devices (Vitrobot, chameleon, and a timeresolved cryoEM device) on various timescales, including grids made within $6 \mathrm{~ms}$ (the fastest reported to date), to interrogate particle behavior at the air-water interface for different timepoints. Results demonstrate that different macromolecular complexes can respond to the thin-film environment formed during cryoEM sample preparation in highly variable ways, shedding light on why cryoEM sample preparation can be difficult to optimize. We demonstrate that reducing time between sample application and vitrification is just one tool to improve cryoEM grid quality, but that it is unlikely to be a generic "silver bullet" for improving the quality of every cryoEM sample preparation.

\section{INTRODUCTION}

Single-particle cryoelectron microscopy (cryoEM) has emerged as a major structural biology technique during the last decade (Kuehlbrandt, 2014). While refined data processing software (Fernandez-Leiro and Scheres, 2017; Punjani et al., 2017; de la Rosa-Trevin et al., 2016) and automated data acquisition (Thompson et al., 2019) have streamlined the technique, sample preparation remains a major bottleneck for many projects. For single-particle cryoEM sample preparation, the specimen is typically spread as liquid film as thinly as possible ( $\approx 20 \mathrm{~nm}$ ) (Rice et al., 2018) before being rapidly vitrified by plunging into a cryogenic liquid such as ethane (Dubochet and Lepault, 1984). The formation of this thin film has commonly been achieved by applying a relatively large sample volume (3-4 $\mu \mathrm{L})$ to a cryoEM grid and then blotting away excess liquid with filter paper. The
cryoEM grid, a $3 \mathrm{~mm}$ diameter metal (commonly copper) disk with square windows, has a support layer (typically amorphous carbon) with small, usually circular perforations ( 1-2 $\mu \mathrm{m}$ diameter) in a regular array. The typical blotting process removes almost all of the liquid applied to the grid, leaving a thin film of sample suspended across the holes in the support where imaging can occur. This procedure was pioneered over 30 years ago by Dubochet and Lepault (1984).

Formation of a thin film using blotting paper followed by vitrification can be achieved through manual and home-built devices, as well as using commercially available devices such as the Vitrobot (Thermo Fisher Scientific), EM GP (Leica Microsystems), and CP3 (Gatan), for which the general concept remains the same as when the method was first conceived. While there can be problems with reproducibility of thin-film formation through a blotting approach it is undeniably successful, resulting 


\section{CellPress OPEN ACCESS}
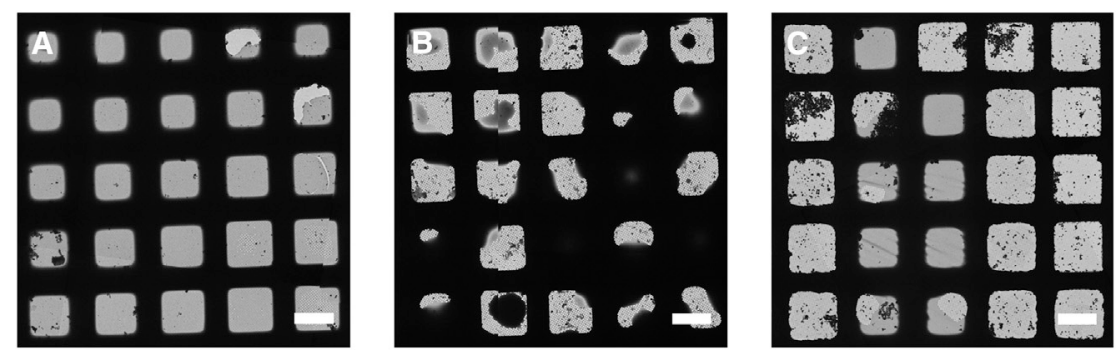

in its application to a broad range of specimens, and it has consequently come to underpin the vast majority of single-particle structures to date.

Over the years, and across different fields of research, it has been shown that the air-water interface (AWI) can be a hostile environment for proteins and macromolecular complexes (Glaeser and Han, 2017; Zhao and Cieplak, 2017; Gerhardt et al., 2014; Wiesbauer et al., 2013). In a typical cryoEM grid preparation both sides of the thin film are exposed to the AWI, creating a very high surface-area-to-volume ratio. Blotting and plunging into cryogens usually takes seconds, during which time the sample can come into contact with the AWI hundreds to thousands of times. Macromolecular complexes and proteins can interact preferentially with and/or denature (either fully or partially) on exposure to the AWI (Taylor and Glaeser, 2008; D'Imprima et al., 2019).

A recent systematic study of particle localization on cryoEM grids prepared with traditional blotting methods by Noble et al. (2018a) has shown that $\sim 90 \%$ of the 46 samples analyzed associate with the AWI, demonstrating that the vast majority of specimens have the potential to be perturbed by the AWI. Recent advancements have led to a greater awareness of variables that can be changed to alter the distribution and behavior of particles on a cryoEM grid. These include the use of grid supports made of different materials such as carbon or gold (Russo and Passmore, 2016), the use of continuous support films (Hurdiss et al., 2016; Russo and Passmore, 2014), affinity grids (Han et al., 2012), the addition of detergents or surfactants (Chen et al., 2019), or reducing the time between sample application and vitrification (Noble et al., 2018b). All of these approaches are linked by a common theme: they either sequester particles away from an AWI or they modulate the properties of the AWI by adjusting chemical properties and surface tension of the liquid film (Glaeser and Han, 2017).

The grid-making process is currently a major focus in the cryoEM field, with a number of approaches in various stages of development, all seeking to improve access, quality, and/or reproducibility of cryoEM sample preparation. The Spotiton system uses an inkjet piezo dispenser to directly deposit samples onto self-wicking grids to create a thin film, and is currently undergoing commercialization (chameleon; SPT Labtech, formerly TTP Labtech) (Razinkov et al., 2016; Wei et al., 2018; Dandey et al., 2018). An alternative open-source approach, the "Shake-it-off," uses an off-the-shelf ultrasonic humidifier to spray small sample volumes onto an electron microscopy (EM) grid and offers a low-cost solution to grid preparation (Rubinstein et al., 2019). The cryoWriter system uses a microcapillary to deposit sample directly on the grid, enabling direct purification and

\section{Structure Resource}

Figure 1. Example Low-Magnification Images of Grids Prepared Using Different Vitrification Devices

Comparison of typical results for (A) Vitrobot, (B) TED, and (C) chameleon (scale bar, $50 \mu \mathrm{m}$ ) as imaged by cryoEM.

vitrification from low volumes of lysate (Arnold et al., 2017; Schmidli et al., 2019). The Vitrojet (CryoSol) uses a pin printing system to deposit small volumes of sample onto the surface of a grid to directly create a thin film in a controlled manner, followed by vitrification with jets of cryogen (Ravelli et al., 2019). Finally, microfluidic spraying devices such as the time-resolved cryoEM device (TED) enable fast dispense-to-plunge times (Kontziampasis et al., 2019) but require larger sample volumes. In this study we focus on the behavior of particles prepared for cryoEM using the Vitrobot Mk IV, TED, and chameleon. Since each of these sample preparation devices exposes particles to different environments, forces, and timescales, we will briefly describe the specifics of each device.

The Vitrobot involves the application of 3-4 $\mu \mathrm{L}$ of sample volume onto an EM grid held in a temperature- and humiditycontrolled chamber. Subsequently it is blotted between two sheets of filter paper, for 3-10 s, removing the vast majority of the sample volume, before the blotting paper is withdrawn and the sample is plunged into the cryogen. Grids can be prepared on a timescale of 5-15 s from sample application, and typical grids will have a gradient containing some areas that are too thick and some that are too thin, with a large number of suitable grid squares for imaging (Figure 1A) (Thompson et al., 2019). While this device has been used to successfully vitrify a wide range of specimens, there is evidence that the irregular pattern of fibers in the filter paper causes non-uniform alterations in surface-to-volume ratio across the grid, and this may be a root cause of the irreproducibility often reported for blotting paperbased vitrification techniques, as well as being detrimental to samples (Armstrong et al., 2020).

The TED was primarily designed to perform time-resolved experiments by rapidly mixing constituents before vitrification on the millisecond timescale. However, in this study we only make use of its ability to deposit a single sample and vitrify it on a very fast timescale ( $\geq 6 \mathrm{~ms}$ ) (Kontziampasis et al., 2019). A conventional EM grid is placed on a plunging arm, which has an adjustable speed within a high-humidity chamber at room temperature. The liquid system (syringes, tubing, and nozzle) is then equilibrated with $\sim 40 \mu \mathrm{L}$ of sample, which is deposited by spraying directly onto the grid as it plunges into the cryogen. A typical experiment requires between 4 and $32 \mu \mathrm{L}$ of sample volume per grid, depending mainly on the liquid flow rate. Exposure time to the AWI is determined by the time of flight for the spray droplets (from nozzle to grid) and the grid plunge time (from spray to ethane). A typical grid has a random droplet pattern, with some thick regions corresponding to the center of a droplet, and thinner edges (which sometimes cover about half of a grid square) where the ice is sufficiently thin for imaging (Figure 1B). With the current design, dispense-to-plunge times can be set from $6 \mathrm{~ms}$ to seconds. 


\section{Structure} Resource

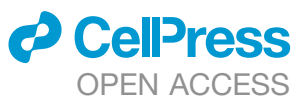

The chameleon is a fully automated instrument that dispenses controlled droplets onto a self-wicking grid as it plunges into the cryogen. Self-wicking grids and $5 \mu \mathrm{L}$ of sample are manually placed into the instrument as input. Workflows guide the user through system setup, preparation of grids, and system cleanup and reporting. Automated assessment of wicking and visual inspection together provides a quality control step prior to cryoEM, allowing the routine preparation of grids with optimal ice thickness. Dispense-to-plunge times range from $54 \mathrm{~ms}$ to a few seconds with typical times in the range of $100-250 \mathrm{~ms}$. A typical grid contains a stripe of approximately 20-40 grid squares with desired ice thickness (Figure 1C).

For this study, we have examined the behavior of three protein systems, apoferritin (480 kDa, O symmetry), mitochondrial chaperone heat-shock protein family D member 1 (HSPD1: mtHSPD1) (408 kDa, C7 symmetry), and Escherichia coli ribosome (30S, 50S, 70S, all C1 symmetry). Apoferritin was chosen because it is a common test specimen in cryoEM, HSPD1 because when prepared using standard cryoEM methods it adopts an extremely preferred orientation, and ribosomes because they are considered to be a very robust macromolecular complex and are also asymmetric, unlike the other two specimens.

\section{RESULTS}

\section{Partitioning of Particles to the AWI}

The speed of grid making has been reported to influence the particle distribution at the AWI, with $\sim 100$ ms showing a change in partitioning and angular orientation relative to slower speeds (Noble et al., 2018b). We used cryoelectron tomography (cryoET) to investigate differences in particle partitioning in the thin ice layer at different time points for various macromolecular complexes, using the Vitrobot, TED, and chameleon (Figure 2A; Videos S1, S2, S3, S4, S5, S6, S7, S8, and S9). Areas for tomogram acquisition were selected without prior investigation of particle distribution in that area, and based upon ice thicknesses that would be deemed most suitable for data collection. We classified particles as partitioned to the AWI based on either a $10 \mathrm{~nm}$ or $20 \mathrm{~nm}$ distance from the AWI. For all three specimen types, on blotted Vitrobot grids (Figure 2B) the majority of particles resided at the $\mathrm{AWI}$, consistent with previous observations (Noble et al., $2018 \mathrm{a}$ ), with an average of $86 \%, 99 \%$, and $80 \%$ of particles associated with the AWI across the apoferritin, HSPD1, and ribosome data, respectively (Figures 2 and S1; Table S1).

To investigate trends in particle distribution on different timescales of vitrification, we used the TED to vitrify grids on "fast" timescales (6-13 ms), and used the TED and chameleon to vitrify grids on "intermediate" timescales (50-200 ms). The majority of the particles partitioned to the AWI on the TED "fast" timescale (apoferritin: $75 \%$ at $11 \mathrm{~ms}$; HSPD1: $89 \%$ at $6 \mathrm{~ms}$; ribosome: $96 \%$ at $13 \mathrm{~ms}$ ), although it should be noted the TED data showed a greater variability compared with Vitrobot data (Figures 2 and $\mathrm{S} 1)$. On the "intermediate" timescale, TED grids of apoferritin (50 ms) and HSPD1 (50 ms) displayed large variability across different tomograms of the same specimen, although the majority of particles interacted with the AWI $67 \%$ and $95 \%$ for apoferritin and HSPD1, respectively). "Intermediate" timescale chameleon grids of ribosome (200 ms) displayed $94 \%$ of sample interacting with the AWI.
The "fast" TED data demonstrate that even on the fastest timescales we could investigate using this device and in thick ice (up to $\sim 180 \mathrm{~nm}$ ), the interaction with the AWI is not eliminated. This is perhaps unsurprising given that calculations suggest that particles will interact 10-100 times with the AWI within $1 \mathrm{~ms}$, and for some proteins this interaction results in sequestering at the AWI (Naydenova and Russo, 2017). It should be noted that TED generally produces thicker ice, especially at faster dispense-to-plunge times, as the TED relies on droplet spreading upon contact with the grid to produce areas sufficiently thin to image (Table S1). For the apoferritin grids prepared using the TED, we observed interesting trends in surface protein aggregates at $11 \mathrm{~ms}$ compared with $50 \mathrm{~ms}$. At $11 \mathrm{~ms}$, small aggregates of $\sim 10-50$ particles were observed, which appeared to be much larger at $50 \mathrm{~ms}$ where they consisted of hundreds of particles. Protein aggregates were only observed at the AWI (Figure S2).

When considering the spraying devices across various timescales and Vitrobot blotting data together, the trend of a reduction in particles at the AWI at faster freezing times holds true for the apoferritin and HSPD1 samples (Figure S1), although more variability is seen in the intermediate timepoints of grids made on the TED. Interestingly, the ribosome data show the opposite trend, with increased partitioning to the AWI at 13 ms compared with the blotted grid. A general observation across all sample preparation techniques (TED, chameleon, Vitrobot) was the presence of asymmetry in particle distribution in some tomograms, i.e., one AWI face was highly populated while the other was not (Figure 2), as previously reported for the Vitrobot and Spotiton (Noble et al., 2018a).

\section{Concentration of Particles}

Our experience with sample preparation has shown that there is a variation in the concentration of the necessary amount of sample required to achieve similar particle numbers in frozen grids when using the Vitrobot, TED, and chameleon. There have been previous studies which have shown that the blotting procedure can increase the apparent concentration of a protein, for example ATP synthase and O3-33, with multiple blotting and the use of detergents significantly affecting the resultant particle concentration on the grid (Snijder et al., 2017; Rubinstein, 2007). In addition to this we wanted to investigate the difference between the concentration of the protein solution used and the resultant concentration on the grid after preparation using the Vitrobot, TED, and chameleon and to compare this with previous estimations using a tomographic approach.

For Vitrobot blotted grids there was a large increase, or concentrating effect, with average 3-, 21-, and 24-fold increases in particle numbers for apoferritin, HSPD1, and ribosomes, respectively (Figure 3). This interesting result demonstrates a previously unreported advantageous sample concentration effect of blotting methods. To interrogate this further, we made a comparison between a theoretical model thin film and the observed data. A model thin film was generated by placing particles representing the actual concentration of sample applied to the grid with randomly generated coordinates (within the confines of the thin film, assuming no concentration change and no affinity for the AWI) (Figure S3). For apoferritin, the model data matched remarkably well the 
A

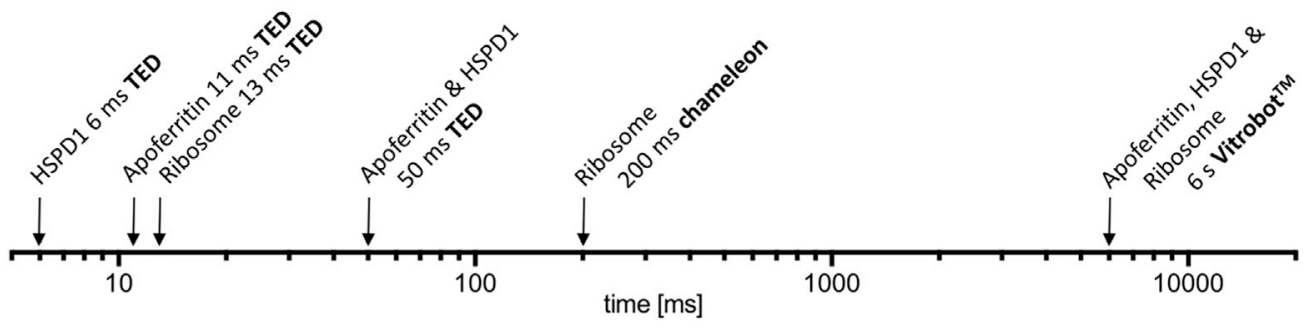

B

C

D Intermediate

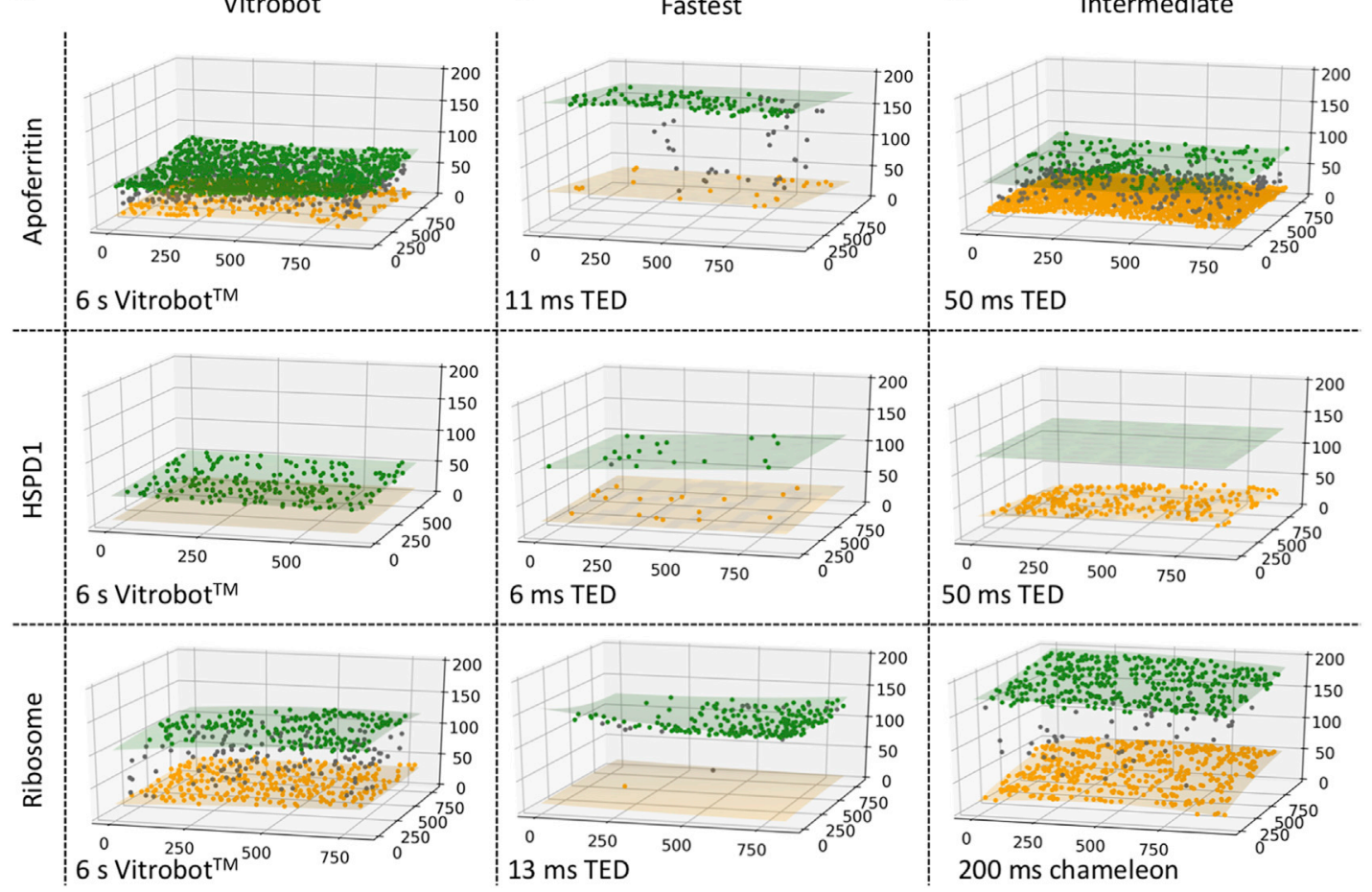

Figure 2. Visualization of Particle Partitioning at AWI Using CryoET

(A) Timescale of grid preparation for tomography samples.

(B-D) Representative tomograms of apoferritin, HSPD1, and ribosome grids prepared at standard blotting speed for Vitrobot (B; time given is Vitrobot "blot time"), (C) fastest time points (TED), and (D) intermediate time points. Green and yellow shaded areas indicate the top and bottom of the AWI, with yellow, gray, and green spheres representing the particle location at the top, middle, and bottom of the ice, respectively. The axis indicates coordinates of the particle location in ice in nanometers. Timescale from sample preparation to vitrification and sample preparation device is shown in the bottom of the box. Full raw data can be seen in Figure S2.

experimental data at distances away from the AWI $(>10 \mathrm{~nm})$, indicating that the concentration effect seen in Vitrobot blotted grids of apoferritin exclusively stems from particles bound to the AWI.

For the TED, we hypothesized that there should be no particle concentration or dilution effects, as the droplets land on the grid without liquid being drawn away as in the case of both the Vitrobot (filter paper) and chameleon (self-wicking grids). Using the TED, at $50 \mathrm{~ms}$ for HSPD1 and apoferritin (and $13 \mathrm{~ms}$ for ribosome), we do indeed see, on average, the number of particles we would expect given the concentration of protein applied. This indicates that there are no significant concentrating effects for TED at these timepoints. However, there is large variability in the $50 \mathrm{~ms}$ apoferritin data compared with the 50 ms HSPD1 data. Interestingly, the "fast" apoferritin and HSPD1 data both show a large depletion of particles
(14- and 7-fold, respectively). Data from the chameleon on the ribosome sample at 200 ms show a substantial concentrating effect (5-fold), but much reduced compared with the Vitrobot data.

\section{Orientation and Angular Distribution of HSPD1}

HSPD1 is known to adopt strong preferred orientation when prepared using standard blot-freezing methods. We examined HSPD1 angular orientation using the TED at 6 and $50 \mathrm{~ms}$, the chameleon at $54 \mathrm{~ms}$, and the Vitrobot (Figure 4). Single-particle datasets for each timepoint and device were collected and combined after pre-processing. Two-dimensional (2D) and three-dimensional (3D) classifications were performed on the combined data to impose the same class selection criteria on all datasets, and the consensus structure was determined. From this, the angular assignments for particles that were frozen 
A

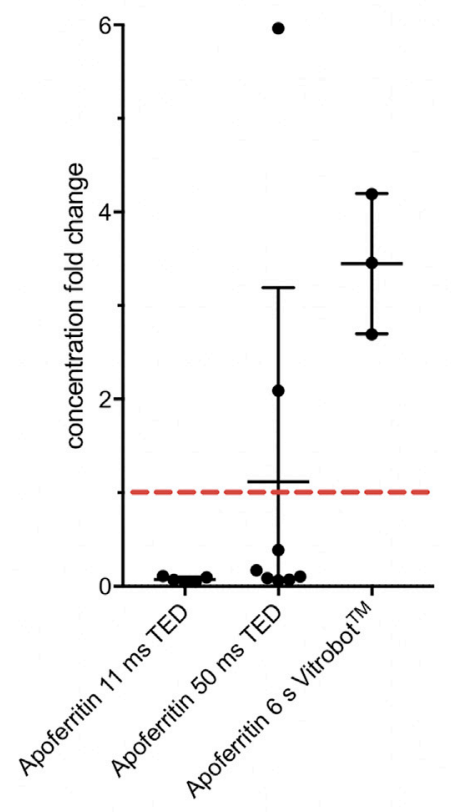

B

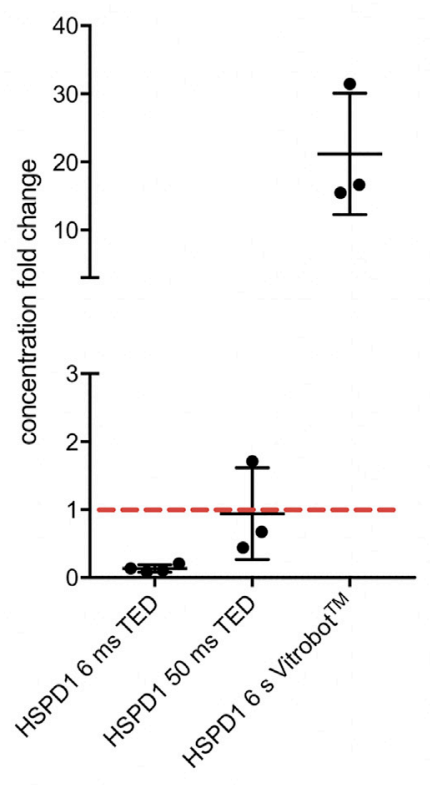

C

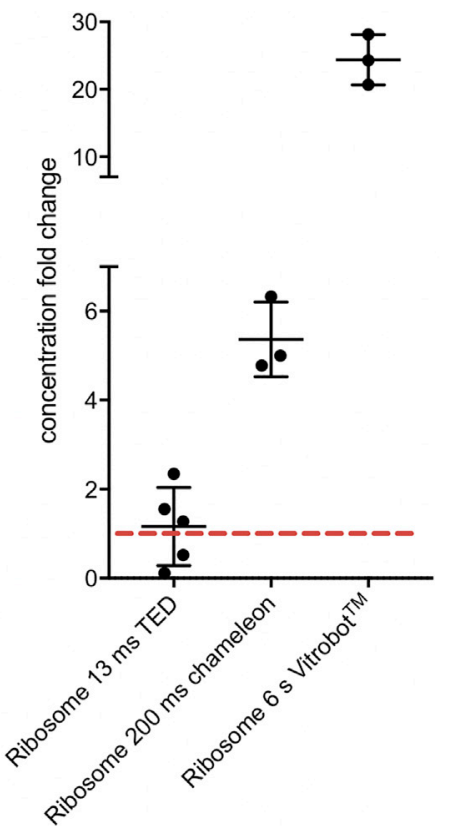

Figure 3. Apparent Change in Protein Concentrations in the Thin Film at Varying Timepoints and Vitrification Devices Particle concentrations in thin film as determined from tomograms for (A) apoferritin, (B) HSPD1, and (C) ribosomes. The solid bars indicate mean \pm SD and dots show individual values. Vitrification device and timescales are labelled. Red line indicates the concentration in solution (applied concentration).

using each device at the specific timepoints were extracted to analyze trends in preferred orientation (Figures 4C and S4).

As expected for HSPD1, strong preferred orientation was seen, with the "top" and "bottom" projections dominating the particle views present in all data collected. The quality of the consensus $3 \mathrm{D}$ reconstruction suffered from the anisotropy of views, as seen in the z-directional Fourier shell correlation (Figures S4B-S4D). The Vitrobot blotted sample (Figure $4 \mathrm{C}$ ) showed the strongest preferred orientation. By increasing the speed of grid making using either the chameleon or TED, broader angular distributions were obtained compared with the standard blotted grid. Reducing the time delay further, from $50 \mathrm{~ms}$ to $6 \mathrm{~ms}$ on the TED, provided further minor improvements in angular distribution, although the data were still dominated by preferred views.

Due to variations between datasets, such as ice thickness and particle number, it is not possible to draw comparisons between the freezing devices used and resolution outcomes. Instead we limit comparisons to the range of angular distributions. For example, the reconstruction from the 6-ms TED data, which had a greater angular distribution, is limited in resolution to approximately $7 \AA$. This is likely due to increased ice thickness compared with the other datasets (Table S1); other reconstructions are likely resolution limited due to low particle numbers or ice thickness (Figure S4).

\section{Orientation and Angular Distribution of Ribosomes}

A sample containing the $305,50 S$, and 705 ribosomes was used to investigate the angular distributions of three related specimens in one dataset to keep as many parameters con- stant as possible (e.g., ice quality). Applying the same approach used to examine HSPD1 angular distribution, we collected single-particle datasets for ribosome samples prepared with TED (13 ms), chameleon (54 and $200 \mathrm{~ms}$ ), and Vitrobot blotted samples (Figures 5 and S5).

The 305 subunit showed a clear correlation between speed of grid preparation and improved angular distribution (Figure 5B). This trend was also present in the $50 \mathrm{~S}$ subunit data, although not as pronounced (Figure 5C). Interestingly, this trend is not present for the full ribosome; instead the greatest angular distribution was observed from grids prepared using the Vitrobot (Figure 5D). Taking the datasets through the processing pipeline, none of the ribosome reconstructions appear to be limited in resolution by angular orientations, and the trends observed in resolution for each of the sample preparation times and methods appear to link most closely to the particle number (Figures S5 and S6).

Consistent with AWI interactions inducing complex dissociation, we observe a number of ribosomal subunits that are resolved at early but not later timepoints. Density for the $50 S$ ribosomal protein L31 is lost in the $70 S$ and $50 S$ ribosome structures in a time-dependent manner (Figures 6B and 6D). In the grids made in $\leq 54 \mathrm{~ms}$ using both TED and chameleon, the L31 subunit is clearly present. However, in those grids made at $200 \mathrm{~ms}$ and $6 \mathrm{~s}$, the L31 subunit is absent within the EM maps when viewed at the same and lower threshold as the fast-plunge structures (Figure 6). During TED, chameleon, or Vitrobot grid preparation, shear forces acting on the sample may vary. Thus, exposure time to the AWI is not the 


\section{CellPress} OPEN ACCESS

\section{Structure}

Resource
A

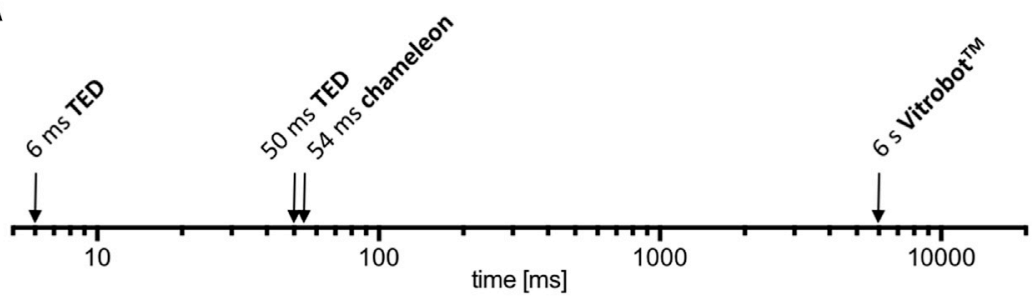

B

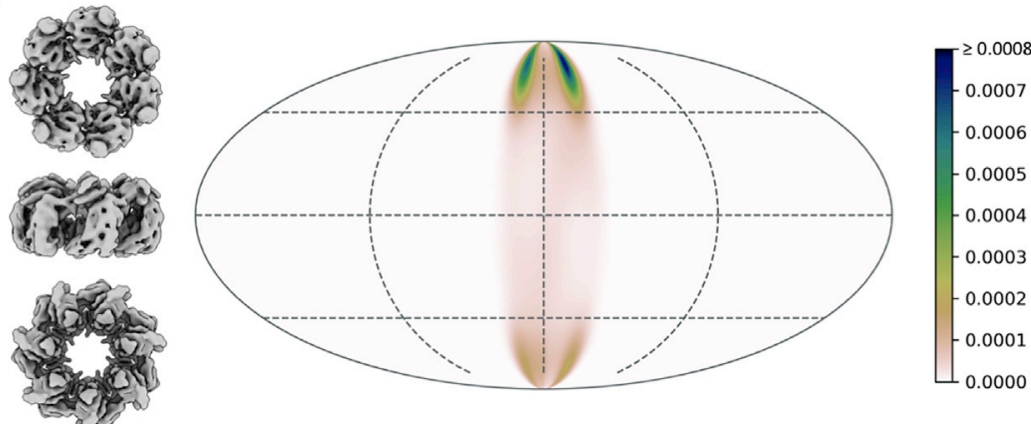

C
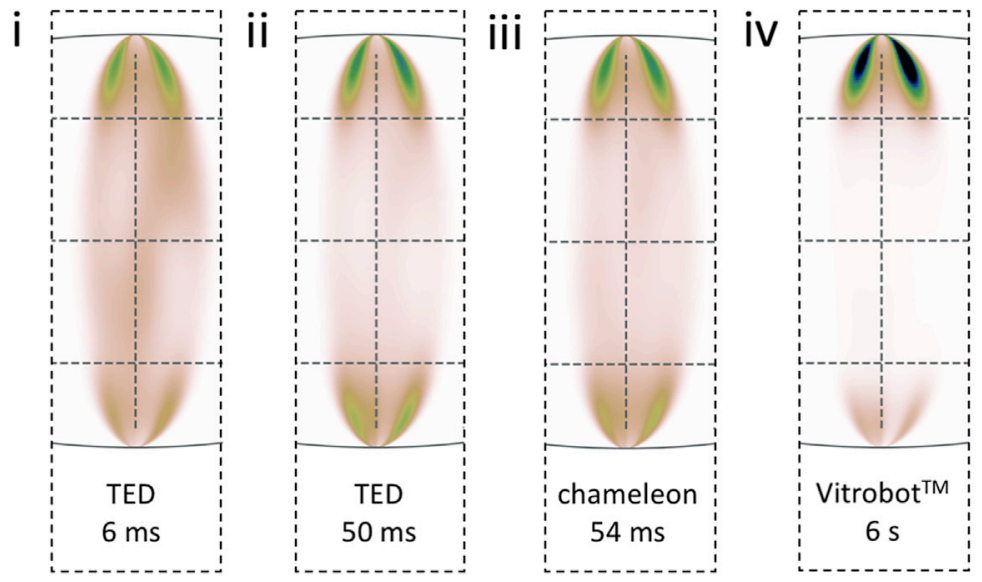

Figure 4. Angular Orientation of HSPD1 over Varying Timepoints and Vitrification Devices (A) Timescale of grid preparation for HSPD1 samples analyzed for angular distribution.

(B) Preferred orientation of HSPD1 of the combined data, showing an angular orientation distribution map (Mollweide projection) of the C7 symmetric reconstruction so that only one-seventh of the area is occupied. Views of HSPD1 on the left show the approximate corresponding orientation, with these data dominated by the top view.

(C) Orientation distribution maps for HSPD1 data collected from samples prepared with TED $6 \mathrm{~ms}$ (i), TED $50 \mathrm{~ms}$ (ii), chameleon $54 \mathrm{~ms}$ (iii), and Vitrobot $6 \mathrm{~s}$ (iv). The normalized probability density function (PDF) approximates the probability to find a particle in a certain orientation. The color scale is the same in (B) and (C), with dark blue representing a high proportion of particles, through green, pink, and white representing no particles in a specific orientation.

area, which may play a role in the relative ease with which these subunits dissociate compared with other subunits (Figure S7).

\section{DISCUSSION}

\section{AWI Partitioning}

The physics of diffusion and AWI interactions cannot be outrun using technology currently available (to the best of our knowledge) for cryoEM sample preparation. Even in the fastest cases of grid vitrification in our study (6 ms) and using different approaches (blotting versus spraying), the majority of particles still partitioned to the AWI. Considering AWI partitioning data from the three specimens we examined, apoferritin, HSPD1, and ribosomes, conflicting lessons can be learned from each. HSPD1 data suggest that the faster the grids are prepared, the fewer particles partition to the AWI (Figures 2 and S1). The ribosome data suggest the precise opposite: the faster the grids are prepared, the more particles partition to the AWI (Figures 2 and S1). The apoferritin data are the most variable and provide the least clear picture across different timescales, which may be partially explained by the propensity of apoferritin to form "rafts" at the AWI (discussed below in Changes in Particle Concentration due to Speed of Grid Preparation).

Overall, altering speed of grid preparation could be one mechanism to influence AWI partitioning, but the effects of this are not linear and are difficult to predict across different specimens. A greater understanding of the factors that may influence partitioning, including specimen polarity, stability, and buffer composition, along with more information about how different specimens respond to the thin-film environment over time, may enable better predictions of specimen behavior prior to freezing in the future. 
A
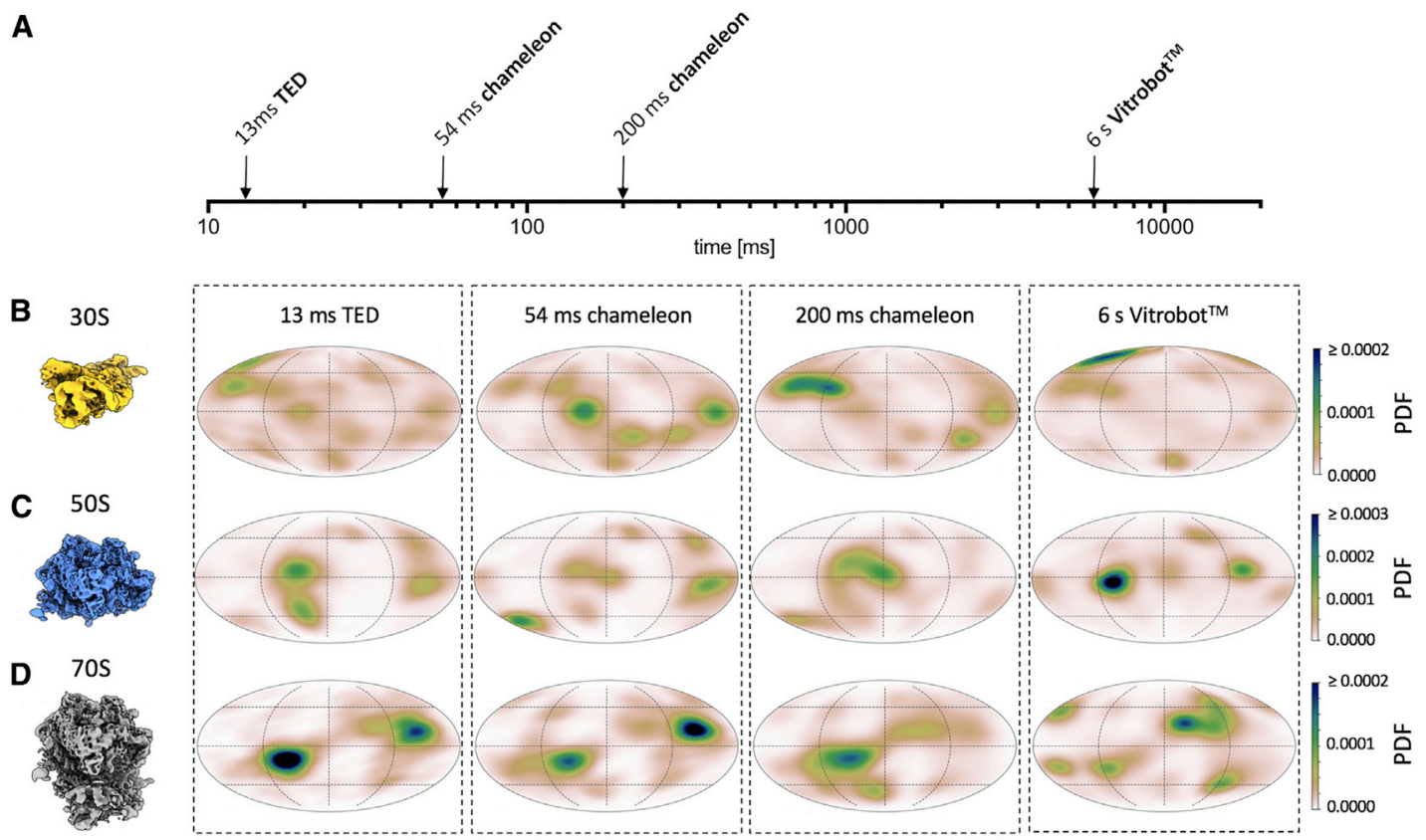

Figure 5. Ribosome Angular Orientation over Varying Timepoints and Vitrification Devices

(A-D) (A) Timescale of grid preparation for ribosome angular single-particle analysis samples. Orientation distribution maps for (B) $30 \mathrm{~S}$, (C) $50 \mathrm{~S}$, and (D) $70 \mathrm{~S}$ samples prepared using stated vitrification device and timescale. As in Figure 4, shown is the normalized probability density function (PDF) in Mollweide projection to approximate the probability to find a particle in a certain orientation.

\section{Changes in Angular Distribution}

Even though most particles could not be prevented from locating at the AWI, small (HSPD1) to very large (30S, 50S ribosome) changes in the angular distribution of particle over tens to hundreds of milliseconds were observed (Figures 4 and 5). Previous work has shown that hemagglutinin from influenza $A$ adopts preferred orientation on the $\sim 100 \mathrm{~ms}$ timescale, slower than HSPD1 but also equilibrating in a single orientation (Noble et al., 2018b; Tan and Rubinstein, 2020). These data suggest that for a given specimen, there may be a fast $(<10 \mathrm{~ms})$ stage when the protein initially partitions to the AWI, followed by a slower stage when the particle explores its energy landscape before settling into a local energy minimum. For some specimens, there may be a distinct orientation (leading to preferred orientation), and for other specimens it may be a variety of orientations (Figure 7). The timescale in this second, slower stage is likely to vary from specimen to specimen.

\section{Particle Damage over Time}

Another factor that must be considered at the AWI is the partial or full denaturation of protein specimens (D'Imprima et al., 2019). The time frame of such denaturation at the AWI is likely to depend on many factors. The ribosome data suggest that denaturation during sample preparation can occur on the timescale of hundreds to thousands of milliseconds. In $70 S$ and 505 ribosomes, the L31 subunit is only present at timepoints $<200 \mathrm{~ms}$, while $30 \mathrm{~S}$ ribosomal subunit $\mathrm{S} 2$ and $50 \mathrm{~S}$ subunit L9 are still present at $200 \mathrm{~ms}$ (Figure 6). In agreement with our data, a recent study has shown that $30 \mathrm{~S}$ ribosomal subunit $\mathrm{S} 2$ is present when grids have carbon support but is dissociated on unsupported grids (Jahagirdar et al., 2020). These data suggest that the timescale of partial denaturation at the AWI is highly specimen dependent and extends into the timeframe accessible by various grid-preparation methods. This timescale suggests that partial denaturation may be an effect of the slower energy landscape exploration, helping to explain how particle orientation can change over longer timescales than partitioning to the AWI takes (Figure 7).

\section{Concentrating Effect of Vitrobot Blotting}

One of the most striking results was the change in concentration due to blotting as compared with spraying (Figure 3). These data clearly demonstrate that for the specimens we have examined, the Vitrobot blotting approach greatly enriches the thin film with particles, consistent with previous studies (Snijder et al., 2017; Rubinstein, 2007), and indeed that the AWI may be responsible for the concentration of particles in the thin film, which in many systems is required to achieve a viable number of particles per micrograph. It should also be noted that the degree of concentration is sample dependent. This may go some way toward explaining the experience of many cryoEM researchers in ascertaining the "right" concentration of protein to use for their system. Adsorption to the grid support may also have a significant impact on apparent particle concentration in the imageable areas, which requires further investigation.

\section{Changes in Particle Concentration due to Speed of Grid Preparation}

Across both TED and chameleon, higher concentrations of specimen were necessary at faster timepoints. However, specifically with TED at the "fast" timescales, a depletion of particles for 
A

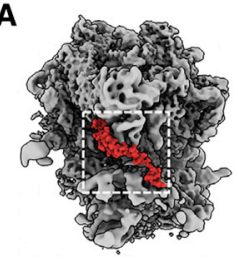

C

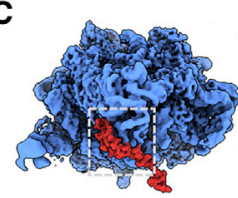

E

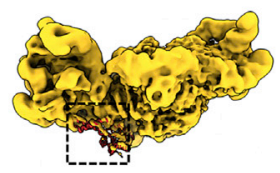

B

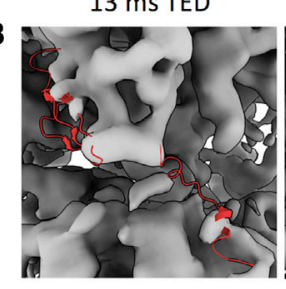

D

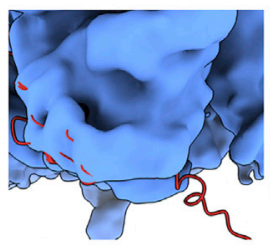

$\mathbf{F}$

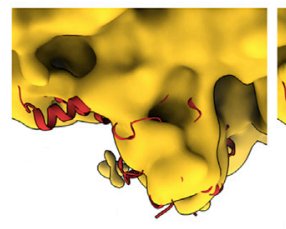

54 ms chameleon
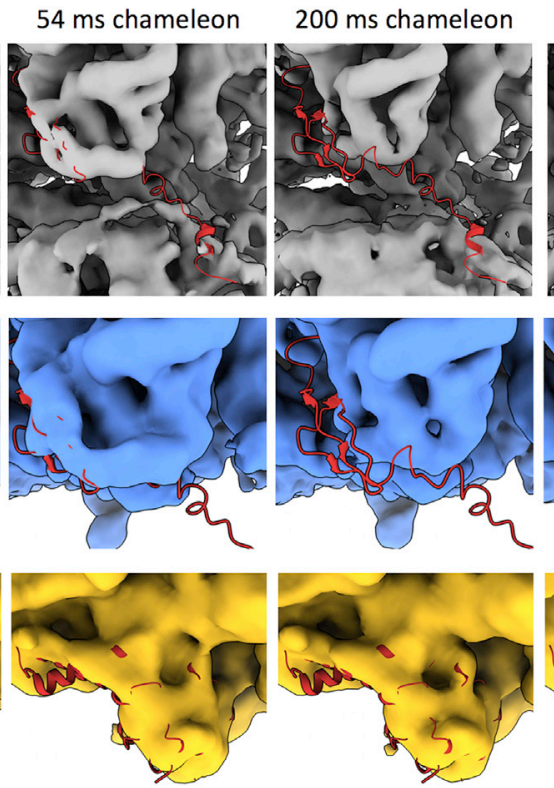

$6 \mathrm{~s}$ Vitrobot $^{\mathrm{TM}}$
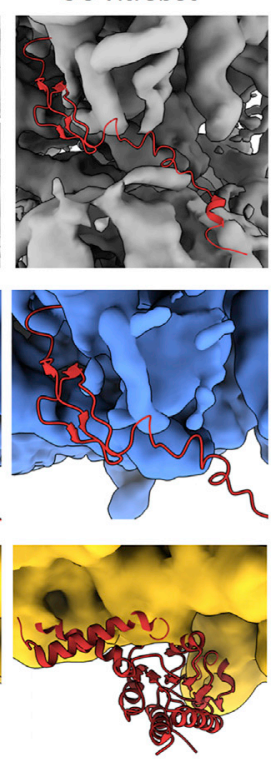

Figure 6. Dissociation of Ribosomal Subunits over Varying Timepoints and Vitrification Devices

(A) Position of ribosomal protein L31 (from PDB: 6OSK) in the 70S ribosome.

(B) Density for L31 in 70 S reconstructions compared between all timepoints. While present in "fast" reconstructions, the density is absent in the $200 \mathrm{~ms}$ or Vitrobot reconstructions.

(C) Position of ribosomal protein L31 (from PDB: 6OSK) in the 50S ribosome.

(D) Density for $\mathrm{L} 31$ in $50 \mathrm{~S}$ reconstructions compared between all timepoints showing the same trend as in (B).

(E) Position of ribosomal protein S2 in the 30S subunit (from PDB: 6O7K).

(F) Density for $\mathrm{S} 2$ in $30 \mathrm{~S}$ reconstructions compared between all timepoints. The $\mathrm{S} 2$ density is missing in the Vitrobot but present in all other reconstructions. $70 \mathrm{~S}$ in gray, $50 \mathrm{~S}$ in blue, and $30 \mathrm{~S}$ in yellow; all maps in (B), (D), and (F) are shown at threshold of $3 \sigma$.

apoferritin and HSPD1 was observed (Figure 3). The apoferritin data from TED display greater variability relative to the other samples, which could be linked to the formation of surface aggregates that were also observed in these data (Figure S2). Surface aggregates, or particle "rafts," may begin to form while the droplet is traveling from nozzle to grid in TED ( $0.5 \mathrm{~ms})$. The size of the aggregates may be time dependent with increases in size occurring at longer timescales. These rafts create locally high concentrations of particles on a single interface (Figures S2Ci and S2Cii). The reason for the preference for just one interface is currently unclear and requires further investigation. Occasionally, large rafts are found in the thin areas chosen for data collection, and are likely also present in the thick regions unsuitable for imaging by transmission EM. This rafting behavior may explain why, on average, the expected number of particles are present in TED 50 ms apoferritin samples, but with large variability in concentration from area to area. A small number of examples of the "raft" effect were also observed for the $13 \mathrm{~ms}$ ribosome grids but not for HSPD1 data, indicating that the presence of rafts is sample dependent while its severity is time dependent. No "rafting" was seen in grids prepared using chameleon or the Vitrobot.

A major unexplained aspect of these data is that for HSPD1 and apoferritin at the "fast" TED time points $(11 \mathrm{~ms}$ and $6 \mathrm{~ms}$ respectively), a large depletion in the concentration of particles compared with slower speeds was observed (Figure 3). We propose the following two hypotheses to explain these observations:
1. The first hypothesis relates to the variability of droplet size, a feature specific to the TED (Figure 1B). The droplets have variable surface-to-volume ratios, so in smaller droplets particles would be more likely to interact with the AWI from the moment the droplet is formed and travels to the grid. If denaturation occurs at this interface, apparent protein concentration would decrease in this droplet and become lower than what would have been observed in larger droplets where the surface-to-volume ratio would favor proportionally fewer AWI interactions. Once on the grid and frozen, smaller droplets are more likely to be imaged, especially at "fast" grid-preparation speeds, as they are more likely to result in thin ice. The larger droplets, which, according to this hypothesis, would contain closer to the expected number of particles, cannot be imaged at these "fast" timepoints because they will result in ice that is too thick. At intermediate grid-preparation timescales, the smaller droplets may have disappeared (due to film thinning) while the larger droplets have thinned to suitable thicknesses. This would explain the apparent depletion of particles observed at faster speeds and the "reappearance" of particles at intermediate timepoints.

2. Particle denaturation over time may also contribute to the observed concentration differences. On TED prepared grids, each frozen droplet has a thick central part that subsequently spreads out into a thin layer at the edge of the 


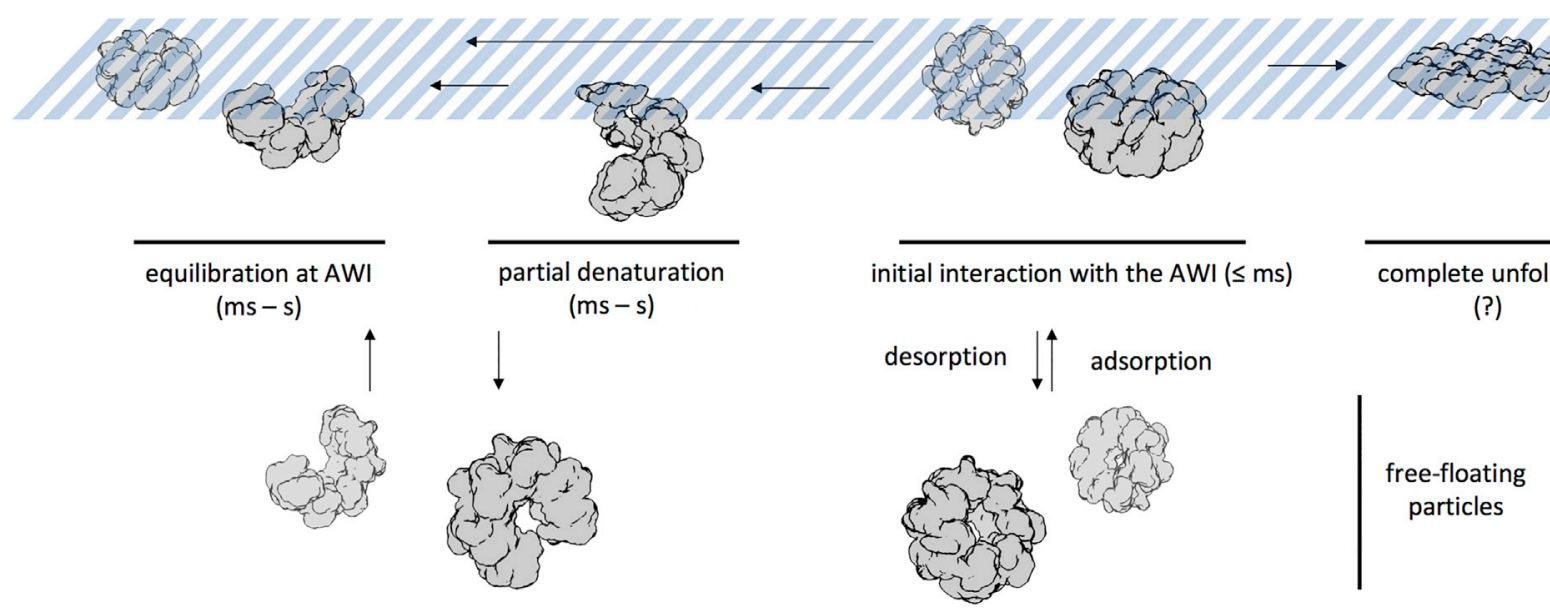

Figure 7. Proposed Model of Protein-AWI Interactions

The initial interaction with the AWI (adsorption) is fast. Equilibration of the protein-AWI system, however, is slower as it involves processes such as desorption, partial, or complete denaturation, which occur on various timescales (milliseconds to seconds) and are thought to be highly protein dependent (desorption from the $\mathrm{AWI}$, rate of unfolding).

droplet. The apparent depletion of particles at the "fast" timepoints may be due to the loss of proteins in the formation of a sacrificial denatured protein layer. Particles could then diffuse from thicker areas in the droplet to repopulate thin, imageable areas, causing the "reappearance" of particles at intermediate timepoints. Theoretical calculations suggest that protein denaturation may occur on a submillisecond timescale (Raffaini and Ganazzoli, 2010) in addition to the longer timescales for partial denaturation seen here (Figure 7).

Formation of a sacrificial layer of denatured protein has been shown for apoferritin (Yoshimura et al., 1994), but the timescale of complete particle denaturation on cryoEM grids remains an open question. There may be alternative explanations for these data, and the hypotheses presented are not mutually exclusive. It is likely that there is interplay between multiple mechanisms on a specimen-dependent basis. It is only with additional information on these trends across many specimens, added to these initial data, that a better understanding of particle behavior in thin films can be achieved.

In conclusion, these data go some way to offering an explanation to those cryoEM researchers who have experienced huge variability in cryoEM sample preparation between biological specimens. General trends indicate that speed may ameliorate some of the adverse effects of the AWI, thus providing a significant improvement in intact or non-preferentially oriented particles. However, this speed may come at the price of a higher required sample concentration, with data suggesting that the faster the grids are prepared, the higher the concentration of protein required. This effect may seem exacerbated given the concentrating effect currently enjoyed when using Vitrobot blotting to prepare samples.

While much is still unknown about the behavior of particle in thin films, a general model can be used to summarize the aforementioned ideas (Figure 7). First, diffusion dictates the rate at which a particle interacts with the AWI. This is an initial fast phase, occurring within $\leq 1 \mathrm{~ms}$ of the thin film forming. Each specimen will then have its own on-off rate and local energy minima at the AWI, determining how likely it is for the protein to disassociate back into bulk solution. Next, negative aspects of the AWI may take place with partial denaturation or dissociation of parts of the molecule, and/or adoption of preferred orientations. However, the timescales of this final equilibrium will likely be highly specimen specific. This model explains why no "silver bullet" has yet been developed to generically tackle cryoEM sample preparation for every specimen. Speed of grid preparation, grid types, use of surfactants, continuous and engineered support, and new grid-making technologies will all have a role to play as the field moves toward the development of generically useful approaches for cryoEM sample preparation, but in the meantime they can be used as individual tools along the research path toward an optimized cryoEM grid.

\section{STAR $\star$ METHODS}

Detailed methods are provided in the online version of this paper and include the following:

- KEY RESOURCES TABLE

- RESOURCE AVAILABILITY

○ Lead Contact

○ Material Availability

O Data and Code Availability

- EXPERIMENTAL MODEL AND SUBJECT DETAILS

- METHOD DETAILS

O Sample Preparation

O Preparation of Blotted Grids

O Fast Preparation of Grids Using the TED

- Fast Preparation of Grids Using the Chameleon

O Fiducial-Less cryoET Data Collection and Processing

O Single Particle cryoEM Data Collection and Processing

- QUANTITATION AND STATISTICAL ANALYSIS 


\section{CellPress} OPEN ACCESS

\section{Structure

\section{SUPPLEMENTAL INFORMATION}

Supplemental Information can be found online at https://doi.org/10.1016/j.str. 2020.07.018.

\section{ACKNOWLEDGMENTS}

We thank Dr. Hao Shao and Dr. Jason Gestwicki (UCSF) for providing the HSPD1 plasmid used in this work. D.P.K. and M.S.C.G. are PhD students on the Wellcome Trust 4-year PhD program in The Astbury Center funded by The University of Leeds. D.J.W. and D.K. were funded by the BBSRC (BB/ P020208/1 and BB/P026397/1, respectively). The FEI Titan Krios microscopes were funded by the University of Leeds (UoL ABSL award) and Wellcome Trust (108466/Z/15/Z). Maps have been deposited within the EMDB; ribosome $30 \mathrm{~S}$ at $13 \mathrm{~ms}, 54 \mathrm{~ms}, 200 \mathrm{~ms}$, and blotted (EMDB: 10871, 10872, 10873, and 10874, respectively), ribosome $50 \mathrm{~S}$ at $13 \mathrm{~ms}, 54 \mathrm{~ms}, 200 \mathrm{~ms}$, and blotted (EMDB: $10875,10876,10877$, and 10878, respectively), ribosome $70 \mathrm{~S}$ at $13 \mathrm{~ms}, 54 \mathrm{~ms}, 200 \mathrm{~ms}$, and blotted (EMDB: 10879, 10880, 10881, and 10882), and HSPD1 at $6 \mathrm{~ms}, 50 \mathrm{~ms}, 54 \mathrm{~ms}$, and blotted (EMDB: 10883, 10884,10885 , and 10886).

\section{AUTHOR CONTRIBUTIONS}

Conceptualization and experimental design: D.P.K., M.S.C.G., D.C.F.M., D.K., M.T., F.S., H.D.W., M.C.D., R.F.T., and S.P.M. Performed experiments: D.P.K., M.S.C.G., D.J.W., M.C.D., and R.F.T. Analysis of data: D.P.K., M.S.C.G., F.S., M.C.D., R.F.T., and S.P.M. Supervision: F.S., R.S.B., H.D.W., R.F.T., and S.P.M. Discussed the data and wrote the manuscript: All authors.

\section{DECLARATION OF INTERESTS}

The authors would like to acknowledge that M.C.D. works for SPT Labtech, the company developing and manufacturing chameleon systems.

Received: May 19, 2020

Revised: July 15, 2020

Accepted: July 29, 2020

Published: August 18, 2020

\section{REFERENCES}

Armstrong, M., Han, B.G., Gomez, S., Turner, J., Fletcher, D.A., and Glaeser, R.M. (2020). Microscale fluid behavior during cryo-EM sample blotting. Biophys. J. 118, 708-719.

Arnold, S.A., Albiez, S., Bieri, A., Syntychaki, A., Adaixo, R., McLeod, R.A., Goldie, K.N., Stahlberg, H., and Braun, T. (2017). Blotting-free and lossless cryo-electron microscopy grid preparation from nanoliter-sized protein samples and single-cell extracts. J. Struct. Biol. 197, 220-226.

Chen, J., Noble, A.J., Kang, J.Y., and Darst, S.A. (2019). Eliminating effects of particle adsorption to the air/water interface in single-particle cryo-electron microscopy: bacterial RNA polymerase and CHAPSO. J. Struct. Biol. X 1, 100005.

D'Imprima, E., Floris, D., Joppe, M., Sánchez, R., Grininger, M., and Kühlbrandt, W. (2019). Protein denaturation at the air-water interface and how to prevent it. eLife 8 , e42747.

Dandey, V.P., Wei, H., Zhang, Z., Tan, Y.Z., Acharya, P., Eng, E.T., Rice, W.J., Kahn, P.A., Potter, C.S., and Carragher, B. (2018). Spotiton: new features and applications. J. Struct. Biol. 202, 161-169.

Dubochet, J., and Lepault, J. (1984). Cryo-electron microscopy of vitrified water. J. Phys. Colloques 45, https://doi.org/10.1051/jphyscol:1984709.

Fernandez-Leiro, R., and Scheres, S.H.W. (2017). A pipeline approach to single-particle processing in RELION. Acta Crystallogr. D Struct. Biol. 73 (Pt 6), 496-502.

Gerhardt, A., Mcgraw, N.R., Schwartz, D.K., Bee, J.S., Carpenter, J.F., and Randolph, T.W. (2014). Protein aggregation and particle formation in prefilled glass syringes. J. Pharm. Sci. 103, 1601-1612.
Glaeser, R.M., and Han, B.-G. (2017). Opinion: hazards faced by macromolecules when confined to thin aqueous films. Biophys. Rep. 3, 1-7.

Goddard, T.D., Huang, C.C., Meng, E.C., Pettersen, E.F., Couch, G.S., Morris, J.H., and Ferrin, T.E. (2018). UCSF ChimeraX: meeting modern challenges in visualization and analysis. Protein Sci. 27, 14-25.

Han, B.-G., Walton, R.W., Song, A., Hwu, P., Stubbs, M.T., Yannone, S.M., Arbeláez, P., Dong, M., and Glaeser, R.M. (2012). Electron microscopy of biotinylated protein complexes bound to streptavidin monolayer crystals. J. Struct. Biol. 180, 249-253.

Van Holde, K.E., and Hill, W.E. (1974). General physical properties of ribosomes. In Ribosomes, M. Nomura and A. Tissiéres, eds. (Cold Spring Harbor Press), pp. 53-91.

Hurdiss, D.L., Morgan, E.L., Thompson, R.F., Prescott, E.L., Panou, M.M., Macdonald, A., and Ranson, N.A. (2016). New structural insights into the genome and minor capsid proteins of BK polyomavirus using cryo-electron microscopy. Structure 24, 528-536.

Jahagirdar, D., Jha, V., Basu, K., Gomez-Blanco, J., Vargas, J., and Ortega, J. (2020). Alternative conformations and motions adopted by 30 S ribosomal subunits visualized by cryo-electron microscopy. bioRxiv. https://doi.org/10. 1101/2020.03.21.001677.

Klebl, D.P., Monteiro, D.C.F., Kontziampasis, D., Kopf, F., Sobott, F., White, H.D., Trebbin, M., and Muench, S.P. (2020). Sample deposition onto cryoEM grids: from sprays to jets and back. Acta Crystallogr. D Struct. Biol. 76, 340-349.

Kontziampasis, D., Klebl, D.P., ladanza, M.G., Scarff, C.A., Kopf, F., Sobott, F., Monteiro, D.C.F., Trebbin, M., Muench, S.P., and White, H.D. (2019). A cryoEM grid preparation device for time-resolved structural studies. IUCrJ 6 (Pt 6), 1024-1031.

Kremer, J.R., Mastronarde, D.N., and McIntosh, J.R. (1996). Computer visualization of three-dimensional image data using IMOD. J. Struct. Biol. $116,71-76$

Kuehlbrandt, W. (2014). The resolution revolution. Science 343, 1443-1444.

Mastronarde, D.N. (1997). Dual-axis tomography: an approach with alignment methods that preserve resolution. J. Struct. Biol. 120, 343-352.

Naydenova, K., and Russo, C.J. (2017). Measuring the effects of particle orientation to improve the efficiency of electron cryomicroscopy. Nat. Commun. 8 , 629-635.

Noble, A.J., Dandey, V.P., Wei, H., Brasch, J., Chase, J., Acharya, P., Tan, Y.Z., Zhang, Z., Kim, L.Y., Scapin, G., et al. (2018a). Routine single particle CryoEM sample and grid characterization by tomography. eLife 7 , https:// doi.org/10.7554/eLife.34257.

Noble, A.J., Wei, H., Dandey, V.P., Zhang, Z., Tan, Y.Z., Potter, C.S., and Carragher, B. (2018b). Reducing effects of particle adsorption to the air-water interface in cryo-EM. Nat. Methods 15, 793-795.

Punjani, A., Rubinstein, J.L., Fleet, D.J., and Brubaker, M.A. (2017). cryOSPARC: algorithms for rapid unsupervised cryo-EM structure determination. Nat. Methods 14, 290-296.

Raffaini, G., and Ganazzoli, F. (2010). Protein adsorption on biomaterial and nanomaterial surfaces: a molecular modeling approach to study non-covalent interactions. J. Appl. Biomater. Biomech. 8, 135-145.

Ravelli, R.B.G., Nijpels, F.J.T., Henderikx, J.M., Weissenberger, G., Thewessem, S., Gijsbers, A., Beulen, B.W.A.M.M., Lopez-Iglesias, C., and Peters, P.J. (2019). Automated cryo-EM sample preparation by pin-printing and jet vitrification. bioRxiv 561, https://doi.org/10.1101/651208.

Razinkov, I., Dandey, V., Wei, H., Zhang, Z., Melnekoff, D., Rice, W.J., Wigge, C., Potter, C.S., and Carragher, B. (2016). A new method for vitrifying samples for cryoEM. J. Struct. Biol. 195, 190-198.

Rice, W.J., Cheng, A., Noble, A.J., Eng, E.T., Kim, L.Y., Carragher, B., and Potter, C.S. (2018). Routine determination of ice thickness for cryo-EM grids. J. Struct. Biol. 204, 38-44.

de la Rosa-Trevin, J.M., Quintana, A., Del Cano, L., Zaldívar, A., Foche, I., Gutiérrez, J., Gómez-Blanco, J., Burguet-Castell, J., Cuenca-Alba, J., Abrishami, V., et al. (2016). Scipion: a software framework toward integration, 


\section{Structure}

\section{Resource}

reproducibility and validation in $3 \mathrm{D}$ electron microscopy. J. Struct. Biol. 195, 93-99.

Rubinstein, J.L. (2007). Structural analysis of membrane protein complexes by single particle electron microscopy. Methods 41, 409-416.

Rubinstein, J.L., Guo, H., Ripstein, Z.A., Haydaroglu, A., Au, A., Yip, C.M., Di Trani, J.M., Benlekbir, S., and Kwok, T. (2019). Shake-it-off: a simple ultrasonic cryo-EM specimen-preparation device. Acta Crystallogr. D Struct. Biol. 75 (Pt 12), 1063-1070.

Russo, C.J., and Passmore, L.A. (2014). Controlling protein adsorption on graphene for cryo-EM using low-energy hydrogen plasmas. Nat. Methods 11 649-652.

Russo, C.J., and Passmore, L.A. (2016). Ultrastable gold substrates: properties of a support for high-resolution electron cryomicroscopy of biologica specimens. J. Struct. Biol. 193, 33-44.

Schmidli, C., Albiez, S., Rima, L., Righetto, R., Mohammed, I., Oliva, P., Kovacik, L., Stahlberg, H., and Braun, T. (2019). Microfluidic protein isolation and sample preparation for high-resolution cryo-EM. Proc. Natl. Acad. Sci. U S A 116, 15007-15012.

Snijder, J., Borst, A.J., Dosey, A., Walls, A.C., Burrell, A., Reddy, V.S., Kollman, J.M., and Veesler, D. (2017). Vitrification after multiple rounds of sample application and blotting improves particle density on cryo-electron microscopy grids. J. Struct. Biol. 198, 38-42.

Tan, Y.Z., and Rubinstein, J.L. (2020). Through-grid wicking enables highspeed cryoEM specimen preparation. bioRxiv. https://doi.org/10.1101/2020. 05.03.075366.

Tang, G., Peng, L., Baldwin, P.R., Mann, D.S., Jiang, W., Rees, I., and Ludtke, S.J. (2007). EMAN2: an extensible image processing suite for electron microscopy. J. Struct. Biol. 157, 38-46.

Taylor, K.A., and Glaeser, R.M. (2008). Retrospective on the early development of cryoelectron microscopy of macromolecules and a prospective on opportunities for the future. J. Struct. Biol. 163, 214-223.
Thompson, R.F., ladanza, M.G., Hesketh, E.L., Rawson, S., and Ranson, N.A. (2019). Collection, pre-processing and on-the-fly analysis of data for high-resolution, single-particle cryo-electron microscopy. Nat. Protoc. 14, 100-118.

Viitanen, P.V., Lorimer, G., Bergmeier, W., Weiss, C., Kessel, M., and Goloubinoff, P. (1998). Purification of mammalian mitochondrial chaperonin 60 through in vitro reconstitution of active oligomers. Methods Enzymol. 290, 203-217.

Wagner, T., Merino, F., Stabrin, M., Moriya, T., Antoni, C., Apelbaum, A., Hagel, P., Sitsel, O., Raisch, T., Prumbaum, D., et al. (2019). SPHIREcrYOLO is a fast and accurate fully automated particle picker for cryo-EM. Commun. Biol. 2, 218.

Wei, H., Dandey, V.P., Zhang, Z., Raczkowski, A., Rice, W.J., Carragher, B. and Potter, C.S. (2018). Optimizing "self-wicking" nanowire grids. J. Struct. Biol. 202, 170-174.

Wiesbauer, J., Prassl, R., and Nidetzky, B. (2013). Renewal of the air-water interface as a critical system parameter of protein stability: aggregation of the human growth hormone and its prevention by surface-active compounds. Langmuir 29, 15240-15250.

Yoshimura, H., Scheybani, T., Baumeister, W., and Nagayama, K. (1994). Twodimensional protein array growth in thin layers of protein solution on aqueous subphases. Langmuir 10, 3290-3295.

Zhang, K. (2016). Gctf: real-time CTF determination and correction. J. Struct. Biol. 193, 1-12.

Zhao, Y., and Cieplak, M. (2017). Proteins at air-water and oil-water interfaces in an all-atom model. Phys. Chem. Chem. Phys. 19, 25197-25206.

Zheng, S.Q., Palovcak, E., Armache, J.P., Verba, K.A., Cheng, Y., and Agard, D.A. (2017). MotionCor2: anisotropic correction of beam-induced motion for improved cryo-electron microscopy. Nat. Methods 14, 331-332.

Zivanov, J., Nakane, T., Forsberg, B.O., Kimanius, D., Hagen, W.J., Lindahl, E., and Scheres, S.H. (2018). New tools for automated high-resolution cryo-EM structure determination in RELION-3. eLife 7, e42166. 


\section{STAR $\star$ METHODS}

\section{KEY RESOURCES TABLE}

\begin{tabular}{|c|c|c|}
\hline REAGENT or RESOURCE & SOURCE & IDENTIFIER \\
\hline \multicolumn{3}{|l|}{ Bacterial and Virus Strains } \\
\hline E. coli BL21 DE3 & Sigma Aldrich & 69450-M \\
\hline \multicolumn{3}{|c|}{ Chemicals, Peptides, and Recombinant Proteins } \\
\hline Horse Spleen apoferritin & Sigma Aldrich & A3660 \\
\hline E. coli ribosome & New England Biolabs & P0763S \\
\hline IPTG & Sigma Aldrich & $367-93-1$ \\
\hline Protease inhibitor cocktail & Calbiochem & $539-134$ \\
\hline Ni-NTA affinity Resin & Generon & NB-45-00042 \\
\hline $10 \mathrm{kDa}$ spin concentrator & Vivaspin (Sartorious) & VS0102 \\
\hline 16/600 Superdex 200 gel filtration column & GE Healthcare & 28-9893-35 \\
\hline Quantifoil 300 mesh CuR 1.2/1.3 grid & Agar Scientific & AGS143-2 \\
\hline \multicolumn{3}{|l|}{ Deposited Data } \\
\hline Ribosome $30 \mathrm{~S}$ (13ms) & From this study & EMDB-10871 \\
\hline Ribosome $30 \mathrm{~S}$ (54ms) & From this study & EMDB-10872 \\
\hline Ribosome $30 \mathrm{~S}$ (200ms) & From this study & EMDB-10873 \\
\hline Ribosome 305 (blot) & From this study & EMDB-10874 \\
\hline Ribosome $50 \mathrm{~S}$ (13ms) & From this study & EMDB-10875 \\
\hline Ribosome $50 \mathrm{~S}$ (54ms) & From this study & EMDB-10876 \\
\hline Ribosome 50S (200ms) & From this study & EMDB-10877 \\
\hline Ribosome 50 S (blot) & From this study & EMDB-10878 \\
\hline Ribosome $70 \mathrm{~S}$ (13ms) & From this study & EMDB-10879 \\
\hline Ribosome 70S (54ms) & From this study & EMDB-10880 \\
\hline Ribosome $70 \mathrm{~S}$ (200ms) & From this study & EMDB-10881 \\
\hline Ribosome $70 S$ (blot) & From this study & EMDB-10882 \\
\hline HSPD1 (6ms) & From this study & EMDB-10883 \\
\hline HSPD1 (50ms) & From this study & EMDB-10884 \\
\hline HSPD1 (54ms) & From this study & EMDB-10885 \\
\hline HSPD1 (blot) & From this study & EMDB-10886 \\
\hline \multicolumn{3}{|l|}{ Recombinant DNA } \\
\hline HSPD1 plasmid & $\begin{array}{l}\text { Dr. Jason Gestwicki \& } \\
\text { Dr. Hao Shao }\end{array}$ & N/A \\
\hline \multicolumn{3}{|l|}{ Software and Algorithms } \\
\hline MotionCor2 & Zheng et al., 2017 & https://emcore.ucsf.edu/ucsf-motioncor2 \\
\hline Imod & $\begin{array}{l}\text { Mastronarde, 1997; } \\
\text { Kremer et al., } 1996\end{array}$ & https://bio3d.colorado.edu/imod/ \\
\hline EMAN2 & Tang et al., 2007 & https://blake.bcm.edu/emanwiki/EMAN2 \\
\hline RELION 3 & Zivanov et al., 2018 & $\begin{array}{l}\text { https://www3.mrc-Imb.cam.ac.uk/relion/index.php?title= } \\
\text { Main_Page }\end{array}$ \\
\hline GCTF & Zhang, 2016 & $\begin{array}{l}\text { https://www2.mrc-Imb.cam.ac.uk/research/locally-developed- } \\
\text { software/zhang-software/\#gctf }\end{array}$ \\
\hline CrYOLO & Wagner et al., 2019 & http://sphire.mpg.de/wiki/doku.php?id=pipeline: window:cryolo \\
\hline ChimeraX & Goddard et al., 2018 & https://www.cgl.ucsf.edu/chimerax/ \\
\hline Mollweide orientation plot & Naydenova and Russo, 2017 & https://www.mrc-Imb.cam.ac.uk/crusso/cryoEF/downloads.html \\
\hline \multicolumn{3}{|l|}{ Other } \\
\hline Vitrobot Mark IV & Thermo Fisher Scientific & $\mathrm{N} / \mathrm{A}$ \\
\hline chameleon & SPT Labtech & N/A \\
\hline TED & Kontziampasis et al., 2019 & $\mathrm{~N} / \mathrm{A}$ \\
\hline
\end{tabular}

e1 Structure 28, 1-11.e1-e4, November 3, 2020 


\section{Structure}

\section{RESOURCE AVAILABILITY}

\section{Lead Contact}

Further requests for resources and reagents should be directed to and will be fulfilled by the Lead Contact, Stephen Muench (s.p.muench@leeds.ac.uk).

\section{Material Availability}

No new reagents were generated in this study.

\section{Data and Code Availability}

The maps have been deposited within the EMDB; ribosome $30 \mathrm{~S}$ at $13 \mathrm{~ms}, 54 \mathrm{~ms}, 200 \mathrm{~ms}$, and blotted (EMDB: 10871, 10872, 10873, and 10874, respectively), ribosome 50S at $13 \mathrm{~ms}, 54 \mathrm{~ms}, 200 \mathrm{~ms}$, and blotted (EMDB: 10875, 10876, 10877, and 10878, respectively), ribosome 70 S at $13 \mathrm{~ms}, 54 \mathrm{~ms}, 200 \mathrm{~ms}$, and blotted (EMDB: 10879, 10880, 10881, and 10882), and HSPD1 at $6 \mathrm{~ms}, 50 \mathrm{~ms}, 54 \mathrm{~ms}$, and blotted (EMDB: 10883, 10884, 10885, and 10886). Raw data is available by contacting the lead author.

\section{EXPERIMENTAL MODEL AND SUBJECT DETAILS}

E. coli BL21 DE3 cells. Culture conditions are described below.

\section{METHOD DETAILS}

\section{Sample Preparation}

Horse spleen apoferritin was purchased from Sigma Aldrich (A3660), and exchanged into $30 \mathrm{mM} \mathrm{HEPES,} 150 \mathrm{mM} \mathrm{NaCl} \mathrm{pH} 7.5$ by ultrafiltration using $100 \mathrm{kDa}$ molecular weight cut-off (MWCO) spin concentrator tubes (Vivaspin, Sartorius). Protein concentration was then determined using absorbance at $280 \mathrm{~nm}\left(\varepsilon_{280}=14,565 \mathrm{~mol}^{-1} \mathrm{~cm}^{-1}, \mathrm{MW}=18.5 \mathrm{kDa}\right.$ and homo-24-mer stoichiometry). For grid preparation, apoferritin was diluted to the target concentration in $30 \mathrm{mM} \mathrm{HEPES,} 150 \mathrm{mM} \mathrm{NaCl} \mathrm{pH} \mathrm{7.5.}$

E. coli ribosome sample was purchased from New England Biolabs (P0763S), provided at a stock concentration of $33 \mathrm{mg} / \mathrm{mL}$ (= approx. $25 \mu \mathrm{M}$ assuming an average molecular weight of $1.34 \mathrm{MDa}$ (Van Holde and Hill, 1974). For grid preparation, ribosomes were diluted to the target concentration using $50 \mathrm{mM}$ HEPES, $8 \mathrm{mM} \mathrm{MgAc} 2,100 \mathrm{mM} \mathrm{KAc} \mathrm{pH} \mathrm{7.5.}$

Mature human mitochondrial heat shock protein family D member 1 (HSPD1) was expressed in E. coli BL21 DE3 and purified based on a modified version previously described protocol (Viitanen et al., 1998). The expression plasmid was kindly provided by $\mathrm{Dr}$ Hao Shao and Dr Jason Gestwicki (UCSF). Competent E. coli BL21 DE3 were transformed with the plasmid using the heat-shock method. Two flasks of $1 \mathrm{~L} \mathrm{~TB}$ media were inoculated with $2 \times 20 \mathrm{~mL}$ overnight culture, incubated for $2.5 \mathrm{~h}$ at $37^{\circ} \mathrm{C}, 200 \mathrm{rpm}$ until $\mathrm{OD}_{600}$ reached 0.8. Expression was induced by adding $250 \mu \mathrm{M}$ IPTG and cells were further incubated for $4 \mathrm{~h}$ at $37^{\circ} \mathrm{C}, 180 \mathrm{rpm}$, then cells were harvested by centrifugation ( $10 \mathrm{~min}, 4000 \mathrm{rpm})$ and stored at $-80^{\circ} \mathrm{C}$.

All purification steps until reconstitution were done on ice or at $4^{\circ} \mathrm{C}$. The cell pellet was thawed on ice and resuspended in $40 \mathrm{~mL}$ lysis buffer ( $50 \mathrm{mM}$ Tris, $500 \mathrm{mM} \mathrm{NaCl}, 10 \mathrm{mM}$ imidazole pH 8), supplemented with $1 \mathrm{mM} \mathrm{PMSF}$ and protease inhibitor cocktail (set V, Calbiochem). The cells were further resuspended with 4 strokes in a dounce homogeniser and lysed with a sonicator (35\% amplitude, $30 \mathrm{sec}$ on/off, $10 \mathrm{~min}$ total). Cell debris was pelleted by centrifugation ( $30 \mathrm{~min}, 17,000 \mathrm{rpm}$ ) and the supernatant applied to $7 \mathrm{~mL}$ packed, equilibrated Ni-NTA resin. The protein-bound resin was washed with $200 \mathrm{~mL}$ lysis buffer and $200 \mathrm{~mL}$ wash buffer (50 mM Tris, $300 \mathrm{mM} \mathrm{NaCl}, 50 \mathrm{mM}$ imidazole pH 8). The protein was eluted with $20 \mathrm{~mL}$ eluting buffer ( $50 \mathrm{mM}$ Tris, $300 \mathrm{mM}$ $\mathrm{NaCl}, 300 \mathrm{mM}$ imidazole $\mathrm{pH}$ 8). To remove the His6-tag, DTT (final concentration $1 \mathrm{mM}$ ) and TEV protease (1.6 mg per $10 \mathrm{~mL}$, $3.2 \mathrm{mg}$ in total) were added and the mixture was incubated for $4 \mathrm{~h}$ at room temperature. The cleavage products were dialysed against $4 \mathrm{~L}$ dialysis buffer $(50 \mathrm{mM}$ Tris, $150 \mathrm{mM} \mathrm{NaCl} \mathrm{pH} \mathrm{7.5)} \mathrm{in} \mathrm{a} 10 \mathrm{kDa}$ MWCO membrane overnight. TEV protease and His6tag were removed by incubation with $3 \mathrm{~mL}$ of preequilibrated Ni-NTA resin for $1 \mathrm{~h}$ in lysis buffer. The flowthrough was collected, $10 \%(\mathrm{v} / \mathrm{v})$ glycerol was added and the protein concentrated to $20-30 \mathrm{mg} / \mathrm{mL}$ in a $10 \mathrm{kDa}$ MWCO spin concentrator (Vivaspin, Sartorius).

For reconstitution into its oligomeric form, $4 \mathrm{~mL} \mathrm{HSPD} 1$ were mixed with $100 \mu \mathrm{L} 1 \mathrm{M} \mathrm{KCl}, 100 \mu \mathrm{L} 1 \mathrm{M} \mathrm{MgAc}_{2}$ and $400 \mu \mathrm{L} 50 \mathrm{mM} \mathrm{Mg}$ ATP (pH 7). The reaction was incubated at $30^{\circ} \mathrm{C}$ for $60-90 \mathrm{~min}$. All following steps were done at room temperature. Precipitate was removed by centrifugation at 13,000 rpm for $10 \mathrm{~min}$ and the soluble fraction was loaded onto a HiLoad 16/600 Superdex 200 gel filtration column (GE Healthcare). Size exclusion chromatography was done in $50 \mathrm{mM}$ Tris, $150 \mathrm{mM} \mathrm{NaCl}, 10 \mathrm{mM} \mathrm{MgCl} 2 \mathrm{pH} 7.7$. The fractions corresponding to oligomeric HSPD1 (as determined by negative stain EM and SDS-PAGE) were collected, concentrated to $10-25 \mathrm{mg} / \mathrm{mL}$ with $10 \mathrm{kDa}$ MWCO spin concentrators, supplemented with $5 \%(\mathrm{v} / \mathrm{v})$ glycerol and frozen in liquid $\mathrm{N}_{2}$.

Protein concentrations of HSPD1 was determined by measuring absorbance at $280 \mathrm{~nm}\left(\varepsilon_{280}=14,440 \mathrm{~mol}^{-1} \mathrm{~cm}^{-1}, \mathrm{MW}=58.2 \mathrm{kDa}\right.$ and homo-7mer stoichiometry). For grid preparation, HSPD1 was diluted to the target concentration in $50 \mathrm{mM}$ Tris, $300 \mathrm{mM} \mathrm{NaCl}$, $10 \mathrm{mM} \mathrm{MgCl} 2 \mathrm{pH} 8$. 
Preparation of Blotted Grids

For specimens prepared by blotting, Quantifoil 300 mesh Cu R 1.2/1.3 holey carbon grids were glow-discharged in a Cressington 208 carbon coater with glow discharge unit at $10 \mathrm{~mA}$ and $0.1 \mathrm{mbar}$ air pressure for $30 \mathrm{~s}$. Grids were prepared using a Vitrobot ${ }^{\mathrm{TM}}$ mark IV (Thermo/FEl) with a blot force of 6 and a blot time of $6 \mathrm{~s}$. The relative humidity $(\mathrm{RH})$ was $\geq 90 \%$ and temperature $20^{\circ} \mathrm{C}$ for ribosome and $4{ }^{\circ} \mathrm{C}$ for apoferritin and HSPD1. Concentrations for Vitrobot ${ }^{\mathrm{TM}}$ grid preparation were $20,0.6$ and $0.8 \mu \mathrm{M}$ for apoferritin $(24 \mathrm{mer})$, HSPD1 (7mer) and ribosome, respectively. The applied sample volume was $3 \mu \mathrm{L}$ for all blotted grids and the liquid ethane was used as cryogen in all cases.

\section{Fast Preparation of Grids Using the TED}

Fast grid preparation using the TED was done as previously described, using gas-dynamic virtual nozzles in spraying mode (Klebl et al., 2020). Quantifoil 300 mesh Cu R 1.2/1.3 holey carbon grids were used after glow-discharge in a Cressington 208 carbon coater with glow discharge unit at $10 \mathrm{~mA}$ and $0.1 \mathrm{mbar}$ air pressure for $99 \mathrm{~s}$. In this TED setup, the droplets are small and fast and the delay between spray and deposition short $(\leq 1 \mathrm{~ms})$. The spray parameters were held approximately constant for all grids, using a liquid flowrate of $8.3 \mu \mathrm{L} / \mathrm{s}$ and an atomizer gas pressure between 1.5 and 2.0 bar. The nozzle design used was slightly different from the one previously described with the distance between liquid channel and nozzle outlet being $95 \mu \mathrm{m}$ instead of $125 \mu \mathrm{m}$. PDMS sprayers were manufactured as previously described. Droplet speeds are high under these conditions (>20 m/s) and the used nozzle-grid distance (during sample application) was low $(7-10 \mathrm{~mm})$. Therefore, to estimate exposure time of the thin film to the AWI, only the time between droplet impact on the grid and freezing was considered. Plunge speeds were measured using a linear potentiometer and the vertical distance between nozzle and liquid ethane surface was $1-3 \mathrm{~cm}$ and the plunge speed was $\leq 3 \mathrm{~m} / \mathrm{s}$. The humidity chamber was at $\geq 80 \% \mathrm{RH}$ and ambient temperature for grid preparation. Concentrations for TED grid preparation were 20,11 and $2.5 \mu \mathrm{M}$ for apoferritin (24mer), HSPD1 (7mer) and ribosome, respectively.

\section{Fast Preparation of Grids Using the Chameleon}

For specimens prepared on the chameleon system, SPT Labtech 300 mesh $\mathrm{Cu}$ R 1.2/0.8 holey carbon self-wicking nanowire grids were used. Variable amounts of glow discharge in a Pelco Easiglow at $12 \mathrm{~mA}, 0.39$ mbar air pressure were used to activate and control the wicking speed. Samples were held at $4^{\circ} \mathrm{C}$ (apoferritin, ribosome) or $24^{\circ} \mathrm{C}$ (HSPD1) until aspiration into the dispenser. Grids were prepared at a $\mathrm{RH}$ between $75 \%$ and $85 \%$ at ambient temperature. The applied sample volume for each stripe is $\sim 6 \mathrm{~nL}$. Concentrations for chameleon grid preparation were 5.5 and $2.5 \mu \mathrm{M}$ for HSPD1 (7mer) and ribosome, respectively.

\section{Fiducial-Less cryoET Data Collection and Processing}

All cryoET was collected in the Astbury Biostructure Laboratory in Leeds on Titan Krios II, using the Gatan K2 direct electron detector operated in counting mode and a Bioquantum energy filter. Data acquisition parameters are listed in Table S1.

Frames were motion-corrected with MotionCor2 (Zheng et al., 2017), stacked using an in-house script and tomograms were reconstructed using back projection in Imod after 4-fold binning to enhance the contrast (Mastronarde, 1997; Kremer et al., 1996). Particles were manually picked using EMAN2 (Tang et al., 2007). Particle positions were then used to locate the AWI. In order to do this, the tomogram was divided into patches in the $\mathrm{x} / \mathrm{y}$-directions (4-16 patches depending on particle concentration). The particles at minimum and maximum Z-height were selected and used to fit a plane (first or second order polynomial, depending on the number of patches and visual inspection of the fit) which corresponds to the upper and lower AWI, respectively. Then, the closest distance was determined between either of the AWIs and each particle. Particles which were at a distance $\leq 10 \mathrm{~nm}$ to an AWI were classed as 'bound' to the AWI. For the majority of tomograms collected, the $10 \mathrm{~nm}$ threshold adequately allowed characterisation of the data, but for tomograms on areas of thick ice $(>80 \mathrm{~nm})$ /where the AWI is not clearly defined, a threshold of $20 \mathrm{~nm}$ was more suitable. Ideal particle behaviour was modelled using the experimentally determined AWIs and randomly generating particle coordinates (number/volume corresponding to the respective concentration) in between the experimental ice layer. Then, distances between modelled particles and AWIs were determined.

\section{Single Particle cryoEM Data Collection and Processing}

All single particle cryoEM data was collected in the Astbury Biostructure Laboratory in Leeds on Titan Krios I, equipped with a FEI Falcon III detector and operated in integrating mode. Data collection parameters are listed in Tables S2 and S3.

All single particle data processing was done in RELION 3 (Zivanov et al., 2018). Micrographs were corrected for beam-induced motion with MotionCor2 and the CTF was estimated using GCTF (Zhang, 2016; Zheng et al., 2017). All further data processing was done as shown in Figures S4 and S5 for HSPD1 and ribosome, respectively. Particles were picked using the general model in crYOLO (Wagner et al., 2019).

All HSPD1 datasets were combined after particle extraction (rescaled to $2.13 \AA$ pixel size). One round each of $2 \mathrm{D}-$ and 3D-classification were used to clean the dataset. Consensus reconstructions with particles from all datasets were generated in $\mathrm{C} 1$ and $\mathrm{C} 7$ symmetry and used to determine angular distributions. Finally, the dataset was split into its original subsets and each subset of particles and used to generate a reconstruction using the assigned angles from the $\mathrm{C} 7$ consensus reconstruction. 


\section{Structure}

Similarly, all ribosome datasets were combined after extraction and subjected to one round of 2D classification to remove 'junk' particles. Then, 3D classification was performed to separate the combined datasets into $70 \mathrm{~S}, 50 \mathrm{~S}$ and $30 \mathrm{~S}$ subsets. Those subsets were cleaned up by an additional round of $2 \mathrm{D}$ classification ( 2 rounds for $30 \mathrm{~S}$ ) and a consensus reconstruction was generated including data from all 4 datasets for the three species (70S, $50 \mathrm{~S}$ and $30 \mathrm{~S})$. The subset for each species was then further split into the original datasets, resulting in reconstructions for $70 \mathrm{~S}, 50 \mathrm{~S}$ and $30 \mathrm{~S}$ for each timepoint.

The maps were visualised using ChimeraX (Goddard et al., 2018). Orientation distributions were visualized using a script adapted from Naydenova et al. (Naydenova and Russo, 2017). The probability density function was estimated using kernel density estimation with a Gaussian kernel at a fixed bandwidth of $10^{\circ}$, wider than the estimated angular accuracy in all cases (to avoid overinterpretation of angular distribution maps).

\section{QUANTITATION AND STATISTICAL ANALYSIS}

CryoEM data collection and processing were performed as described in single particle data collection and processing sections of the Method Details using RELION3, MotionCor2, GCTF and crYOLO as detailed in the Key Resources Table. 